\title{
Quaderni
}

QUADERNI Communication, technologies, pouvoir

71 | Hiver 2009-2010

Le changement climatique : les résistances à

l'adaptation

\section{Les adaptations au changement climatique ou la re- découverte des acteurs et des territoires}

\section{Martine Tabeaud}

\section{(2) OpenEdition \\ Journals}

\section{Édition électronique}

URL : http://journals.openedition.org/quaderni/522

DOI : $10.4000 / q u a d e r n i .522$

ISSN : 2105-2956

Éditeur

Les éditions de la Maison des sciences de l'Homme

Édition imprimée

Date de publication : 5 janvier 2010

Pagination : 7-25

Référence électronique

Martine Tabeaud, «Les adaptations au changement climatique ou la re-découverte des acteurs et des territoires », Quaderni [En ligne], 71 | Hiver 2009-2010, mis en ligne le 05 janvier 2012, consulté le 21 décembre 2020. URL : http://journals.openedition.org/quaderni/522 ; DOI : https://doi.org/10.4000/ quaderni.522 


\section{$D$ ossier}

\section{les adaptations}

au changement climatique ou

la re-découverte

des acteurs et des territoires
En ce début du XXI ${ }^{e}$ siècle, le paradigme sociopolitique communément admis est que le réchauffement climatique contemporain, à $90 \%$ lié aux activités humaines ${ }^{1}$, implique une « lutte $^{2}$ » combinant :

- l'atténuation de l'amplitude du réchauffement c'est-à-dire une « intervention anthropique pour réduire les sources ou augmenter les puits de gaz à effet de serre » (GIEC),

- l'adaptation soit le fait d'« ajuster des systèmes naturels ou des systèmes humains face à un nouvel environnement ou un environnement changeant. L'adaptation aux changements climatiques indique l'ajustement des systèmes naturels ou humains en réponse à des stimuli climatiques présents ou futurs ou à leurs effets, afin d'atténuer les effets néfastes ou d'exploiter des opportunités bénéfiques » (GIEC).

\section{Martine Tabeaud}

Professeur Université Paris 1 UMR CNRS ENEC les répercussions graves voire irréversibles du
Ce double défi se justifierait car d'une part. une réduction immédiate et importante des émissions de gaz à effet de serre (GES) permettrait d'éviter réchauffement (au-delà de $+3^{\circ} \mathrm{C}$ ). Cette hausse (un peu moins de $1^{\circ} \mathrm{C}$ de plus aujourd'hui qu'en 1850) est un enjeu pour tous les humains et dans de nombreuses régions du monde le changement se fait déjà sentir. D'autre part, compte tenu de la durée de vie des GES atmosphériques et des réactions différées des éléments du système planétaire, le réchauffement est inéluctable pour plusieurs décennies à venir. Les estimations les plus optimistes du quatrième rapport d'évaluation du GIEC tablent sur une fourchette d'augmentation d'ici à 2100 allant de $1,8^{\circ} \mathrm{C}$ à $4^{\circ} \mathrm{C}$ par rapport à $1990^{3}$.

Ces perspectives imposent des actions impliquant 
de très nombreux et très divers acteurs. Une liste non exhaustive pourrait comprendre : les organismes internationaux (PNUE, OMC, OMS OMM, etc.), les groupes d'États (OPEP, PED, UE), les États, les régions, les communes, les syndicats intercommunaux, les ONG (associations environnementalistes, corporatistes ou territorialisées), les partis politiques et les syndicats, les banques, le monde des finances et des assurances, les chercheurs (depuis ceux qui représentent leur pays dans le panel intergouvernemental du GIEC jusqu'aux indépendants, de toutes disciplines : économistes, physiciens, sciences sociales, etc.), les entreprises multinationales (pétroliers, multinationales de l'environnement, BTP, entreprises du secteur de l'énergie et des transports, etc.), les PME PMI, la grande distribution, les transports, les médias (TV, radios, presse écrite, Internet, etc.) et enfin les citoyens (consommateurs, habitants, électeurs, touristes, etc.) Il apparaît d'emblée que ces acteurs n'interviennent pas tous aux mêmes échelles (du local au mondial) et que leurs intérêts peuvent converger comme diverger sur le choix et la manière de mener des actions curatives et préventives. D'autre part chaque acteur peut avoir des comportements schizophrènes.

C'est ce positionnement des acteurs entre adaptation et atténuation que nous allons essayer d'interroger au cours des vingt dernières années pour dégager ce que pourrait être des pratiques d'adaptation au changement climatique.

\section{La montée au ciel : à climat planétaire, réponse internationale au nom de l'humanité}

Comme l'air est mobile, ce que chacun observe avec le vent, comme la mobilité de l'atmosphère ne s'arrête pas aux frontières, ce qu'a largement souligné Tchernobyl, comme les gaz ayant entraîné le réchauffement sont présents partout, ce que les glaces de l'Antarctique montrent, les réponses au réchauffement planétaire doivent être mondiales. Tel fut sans doute le paradigme fondateur.

\section{Les acteurs politiques de la mondialisation font le choix de l'atténuation}

Dès 1989, le changement climatique apparaît à l'ordre du jour de la réunion du G7 à Paris. Le sujet quitte le champ scientifique pour le champ politique. À l'Assemblée générale des Nations Unies, il est présenté comme un « enjeu commun de l'humanité ». Une nouvelle étape est franchie avec la Convention Cadre des Nations Unies sur le Changement Climatique (CCNUCC) adoptée à Rio de Janeiro en 1992 qui reconnaît l'importance de protéger le climat; la convention est ratifiée en 1995 par 127 pays. Puis, le Protocole de Kyoto, signé lors de la troisième conférence des Parties (à la CCNUCC) en décembre 1997, fait de l'atténuation un engagement juridique qui impose des limites d'émissions de GES aux pays industrialisés, avec une réduction moyenne de 5,2\% par rapport aux niveaux de 1990, sur la période 2008-2012. La ratification par la Russie en 2004 a permis l'entrée en vigueur du Protocole en 2005. En 2008, 181 pays ont ratifié ce Protocole qui les place clairement dans une stratégie d'atténuation des émissions de GES pour limiter la hausse thermique.

L'objectif de la CCNUCC est explicite : « stabiliser les émissions pour ne pas dépasser un certain seuil de dangerosité » (article 2). L'adaptation au 
changement climatique est mentionnée de façon secondaire dans un seul article de la CCNUCC (article 2 " atteindre ce (un niveau de concentration de GES) niveau dans un délai suffisant pour que les écosystèmes puissent s'adapter naturellement aux changements climatiques », et il ne s'agit pas des sociétés humaines ! De même, le mot « adaptation » est prononcé dans 4 articles du protocole de Kyoto, sans en constituer l'élément essentiel ${ }^{4}$ :

\section{Les militants "écolo " et les scientifiques ont participé aux orientations des politiques}

À partir des années 1970, les idées des ONG environnementalistes se sont fait entendre de plus en plus fort au point d'infléchir les politiques de nombreuses institutions. En vingt ans, elles sont devenues incontournables. À la conférence de Rio en 1992 on comptait 2400 représentants d'ONG pour 108 chefs d'État et à Johannesburg, en 2002, il y avait 7000 représentants d'ONG. Le Grenelle de l'environnement leur a fait une grande place en France en 2007. C'est la preuve que les ONG internationales de l'environnement (WWF, Greenpeace etc.) sont des acteurs efficaces. Elles sont très puissantes et possèdent des ressources supérieures à celles de bien des États. Elles ont des millions de membres. Elles peuvent agir comme courroies de transmission entre les organisations internationales et les gouvernements, comme médiatrices entre États, comme consultantes ou comme groupes de pression en faveur de grandes causes environnementales, très universelles par définition, (« action rapide et concrète en faveur de la protection du climat. " WWF, «Faites un geste pour la planète » WWF, "Protégeons la biodiversité », Greenpeace....).
Leur capacité à diffuser leur message vite et partout en fait des acteurs réactifs. Elles revendiquent plus de poids dans les décisions climatiques : droit de participation dans les décisions, droit d'accès aux négociations. La question récurrente concernant ces ONGE est celle de leur représentativité. Reflètent-elles les perspectives de certaines sociétés et cultures dominantes ou bien des aspirations universelles ? Sur le climat, leurs mots d'ordre ont clairement situé le débat à l'échelle de la planète, pour les générations futures, pour la survie de l'humanité... Pour insister sur cet avenir noir commun à tous, leur terminologie a substitué à « pays riche » celle de pays « mal développés » (vs « sous-développés »).

En cela, elles réinterprètent à leur manière les résultats de chercheurs issus des sciences du climat. En 2007, l'attribution du Prix Nobel de la Paix conjointement à Al Gore et au GIEC a souligné l'importance du panel intergouvernemental, le GIEC. Il a été créé en 1988, en marge du PNUE et de l'OMM, qui le financent. Il rassemble des experts nommés pour 5-6 ans par les gouvernements. Des rapports de synthèse périodiques $(1990 ; 1995 ; 2001 ; 2007)$ ont pour fonction d' "évaluer les informations d'ordre scientifique, technique et socio-économique nécessaires pour comprendre les fondements des risques liés au changement climatique d'origine humaine, cerner les conséquences possibles» (introduction au rapport 1990). Trois groupes de travail ont été mis en place : le Groupe de travail I évalue les aspects scientifiques du système climatique et de l'évolution du climat, le Groupe de travail II s'occupe de la vulnérabilité des systèmes socio-économiques et naturels aux changements climatiques, des conséquences 
négatives et positives de ces changements et des possibilités de s'y adapter, le Groupe de travail III évalue les solutions envisageables pour limiter les émissions de GES ou atténuer de toute autre manière les changements climatiques. Avec ces rapports périodiques, le GIEC définit en quelque sorte la teneur des problèmes climatiques. Et cela d'autant plus qu'il rédige aussi des « Résumés pour décideurs » approuvés à l'unanimité ligne par ligne par les représentants des pays. Dans les premiers rapports de 1990 et 1995 , c'est le groupe I, celui des climatologues qui a eu la plus forte audience et dont les conclusions sur le réchauffement attendu pour 2049, 2099, etc. ont été reprises le plus largement. D'où l'accent mis sur l'atténuation du réchauffement. Certes, l'adaptation devait être traitée dans le groupe II (WG II), mais il s'est concentré sur l'évaluation des impacts. En dehors des chercheurs des sciences dures, les économistes ont acquis un poids de plus en plus grand dans le GIEC car aucun futur ne peut être modélisé sans prospectives démographiques, choix de production, de consommation, etc. Les macro-économistes s'efforcent d'intégrer aux modèles les mécanismes de marché qui conditionnent les émissions de GES. Leur travail se heurte à de nombreux débats (efficacité et puissance du marché, environnement et équité, etc.).

Mettre l'adaptation en avant, signifie au minimum bouleverser la hiérarchie d'influence des groupes du GIEC et au mieux intégrer toutes les disciplines, y compris les sciences humaines et sociales. C'est aussi une forme de contestation des méthodes et notamment de l'importance accordée à la modélisation numérique. Pour certains chercheurs du Sud la modélisation est un
« langage du Nord» qui a eu ses mérites mais ne peut plus suffire. Les critiques s'expriment aussi sur l'échelle macro qui permet que le GIEC « efface le passé, naturalise le présent et globalise le futur $»^{5}$. Cette vision admet l'état actuel comme neutre, alors qu'il est hérité d'un long processus historique. Quant à l'appréhension planétaire des GES, elle gomme les disparités régionales. Avec les années, un nombre de plus en plus grand de chercheurs des sciences naturelles, appliquées, sociales et humaines revendiquent une autre approche, où l'adaptation aurait plus de place.

\section{L'UE à la recherche du leadership mondial de l'environnement}

La lutte contre le réchauffement a pu offrir une possibilité d'asseoir un leadership sur la scène internationale. En la matière, l'UE cherche à être un chef de file. S'appuyant sur sa bonne réputation environnementale elle a tenté de diffuser des idées comme le principe de précaution ${ }^{6}$, Elle a occupé une place très importante dans les négociations sur le Protocole de Kyoto. Plus tard, le retrait américain de 2001 lui a fourni un argument identitaire, l'occasion d'assumer la pole position environnementale de la planète. Le profil énergétique de l'Europe lui permet de prôner des politiques d'atténuation poussées par d'influents écologistes. Dans « Kyoto », l'UE a un engagement commun de réduction de $8 \%$, mais certains pays se montrent plus engagés que d'autres. Deux locomotives servent de fer de lance. L'Allemagne s'est fixé un objectif de réductions d'émissions de $40 \%$ d'ici à 2020 , plus ambitieux que celui posé dans l'Annexe B du Protocole (-21\%) afin de devenir une société moderne à énergies renouvelables et au très haut 
niveau d'efficacité énergétique. En France, avec le « Facteur 4 », « la lutte contre le changement climatique est une priorité de la politique énergétique ». L'option d'atténuation est facilitée par l'importance du nucléaire qui ne dégage pas de GES. En redonnant à ce secteur une virginité environnementale perdue après Tchernobyl, cela permet de défendre les intérêts économiques de l'entreprise Areva. Par ailleurs, la lutte contre le réchauffement par l'atténuation fournit une solution pour tous qui ne peut que plaire au pays de la déclaration universelle des droits de l'homme.

Pour l'après Kyoto, L'UE persiste et signe considérant qu'il est essentiel que l'accord à venir limite le réchauffement planétaire à $+2^{\circ} \mathrm{C}$, pour se protéger des changements irréversibles, voire catastrophiques. Le respect d'un tel plafond impose que les émissions mondiales de GES soient réduites de plus de $50 \%$ d'ici 2050 (ref. 1990). Les chefs d'États et de gouvernement de l'Union Européenne se sont déclarés favorables à un objectif de - $30 \%$ d'ici 2020, pour autant que d'autres pays développés s'engagent à atteindre des réductions comparables et que les pays émergents contribuent selon leurs capacités respectives. Ils ont également décidé une réduction d'au moins $20 \%$ sur la même période, quoique fassent les autres pays, afin de commencer à transformer l'Europe. La politique européenne affiche donc un fort volontarisme en faveur de l'atténuation.

\section{Les critiques : un ramassis hétérogène}

Durant les vingt dernières années, de nombreuses voix se sont élevées pour critiquer le choix de l'atténuation.
Depuis toujours, les Pays en développement (PED) sont opposés à la logique d'atténuation considérée par eux comme une entrave majeure à leur développement. Ils tentent de se faire entendre sur des actions de transfert technologique en mettant l'accent sur des garanties de financement. Cette position n'a pas toujours les mêmes motivations. Si elle apparaît légitime pour les pays les plus pauvres et les petits États insulaires, pour les pays émergents et les pays producteurs de pétrole, il s'agir surtout de ne pas s'engager sur des objectifs de réductions d'émissions alors que la croissance de leurs émissions rendrait légitime leur participation à l'effort international.

Malgré les progrès de la modélisation, les scenarii prospectifs proposent des fourchettes jugées trop larges sur le devenir du climat, ce qui ne devrait pas changer avec la complexification des éléments pris en compte. Ces incertitudes poussent à un certain scepticisme. L'effet a été renforcé par des propositions de solutions sur les symptômes relevant de la géoinginierie à l'atténuation du type Docteur Mabuse : ensemencements soufrés des nuages de toute la planète reproduisant les éruptions volcaniques, aspersion des océans avec de la poudre de fer (James Hansen, John Schellnhuber, Paul Crutzen 7 , etc.). Au lieu de rassurer, ces perspectives coûteuses et aux conséquences mal maîtrisées ont fait craindre une dérive vers la climatocratie.

Plus généralement, l'échec sur l'atténuation du marché international du carbone type Kyoto a eu un effet démobilisateur. Comme la moitié des GES émis proviennent de pays n'ayant pas ratifié Kyoto ou sans engagement jusqu'en 2012, les quantités émises dans le monde se sont ac- 
crues de 3,4 \% par an depuis 2001.L'atténuation se heurte là aux besoins accrus d'énergie des pays émergents (Chine, Inde) et à la volonté de ne pas entrer en récession (États-Unis) avec la décroissance.

Sous couvert d'unité de façade, il est clair que les pays fonctionnent en ordre dispersé : c'est le cas dans l'UE. Les stratégies d'indépendance énergétique conduisent à des choix non partagés comme celui du nucléaire choisi en France et abandonné en Allemagne, comme les accords bilatéraux avec Gazprom, via des groupes pétroliers en Italie ou en Allemagne.

Dans un contexte économique morose, avec un fort taux de chômage dans un grand nombre de pays, des stratégies à deux, trois voire quatre générations apparaissent trop lointaines pour être crédibles.

Le succès de l'atténuation s'explique car la globalisation de la lutte contre l'effet de serre a l'avantage de placer l'humanité toute entière dans une positon simple de responsable/victime : responsable des émissions et victime des conséquences de ce dégazage. Mais avec ce type de raisonnement, les sociétés sont indifférenciées : toutes ont pollué au même niveau, tous les territoires $^{8}$ sont équivalents face aux nouveaux risques.

Les pays riches assument a minima leur responsabilité historique et assurent la pérennité de leur suprématie internationale, avec une aide là encore a minima aux pays qui n'ont pas participé aux modifications de l'atmosphère et doivent aussi participer à l'effort collectif.

\section{Le retour sur Terre : à problème planétaire, réponse adaptée aux lieux}

Même si des signes précurseurs (comme la création de l'Observatoire National sur les Effets du Réchauffement Climatique en France en 2001), le « déclic » concernant l'adaptation date de 2006 et du rapport Stern ${ }^{9}$ qui quantifie les coûts de la lutte contre le changement climatique. Pour une élévation de la température moyenne comprise entre 3 et $4{ }^{\circ} \mathrm{C}$, les coûts supplémentaires d'adaptation des infrastructures et des bâtiments pourraient déjà atteindre 1 à $10 \%$ du total des investissements réalisés dans la construction par les pays de l'OCDE. Si rien n'est fait pour empêcher une forte hausse des températures, les mesures d'adaptation verront leur coût monter en flèche (à long terme $20 \%$ de la performance économique mondiale) et leur efficacité relative diminuer. Si la communauté internationale veut maitriser ces coûts, elle doit se fixer des objectifs d'adaptation Agir dès maintenant représente des économies pour l'avenir. Le rapport bien que contesté a donc mis l'adaptation au devant de la scène.

\section{De la vulnérabilité des pays pauvres aux problèmes des pays riches}

Quelques lieux emblématiques ont catalysé les discours sur les conséquences de la hausse du niveau marin sur des milieux naturels fragiles (les îles coralliennes) ou très peuplés (deltas du Gange Brahmapoutre) et sur la fonte de la banquise mettant en péril une civilisation, celle des Inuits. La peur du déluge, en quelque sorte, a ouvert les yeux sur le fait que les humains ne sont pas égaux face aux risques climatiques. Les plus soumis au climat et les plus pauvres sont les plus 
vulnérables. Cette prise de conscience s'est traduite pour les signataires du Protocole de Kyoto par la création à Marrakech, d'un Fonds spécial pour financer des actions dans les PED, complété ensuite en 2004 et 2005 par le " Buenos Aires Programme of Work on Adaptation Response Measures ». Le montant de ce fond est de l'ordre de 600 millions d'euros (à comparer avec les 470 milliards mis par le gouvernement allemand pour sauver ses banques, 360 en France, 200 aux Pays Bas, 200 en Italie, etc. !). L'OCDE, depuis 2005, avec le Programme de Nairobi, insiste sur les incidences des changements climatiques, la vulnérabilité et l'adaptation. Il comprend neuf thématiques mises en œuvre depuis $2007^{10}$. Bien que sans ressources, il fournit des informations sur l'utilisation la plus efficace des ressources financières disponibles. Il a pour objectif d'aider toutes les parties à la CCNUCC, notamment les PED, à mieux comprendre et évaluer les impacts, la vulnérabilité et l'adaptation aux changements climatiques. À partir d'octobre 2007, 87 organisations intergouvernementales et $\mathrm{ONG}$ ont offert d'y participer. Il s'agit là d'une première avancée sur le plan international. Ban Ki Moon, le 29 septembre 2008 à l'assemblée des Nations Unies l'a confirmé : « Des pays tels que le Bangladesh, Cuba, la Jamaïque, Madagascar et les Philippines ont montré que des bâtiments bien conçus, une utilisation appropriée des terrains, une formation du public, une préparation des communautés et des systèmes d'alertes efficaces peuvent réduire l'impact des pires événements météorologiques ». Cette re-découverte des lieux et des inégalités de vulnérabilités qui font le risque s'est ensuite propagée aux pays riches. Le risque climatique grâce aux vulnérabilités redevient pluriel. Tant qu'il se réduisait à l'aléa, la solution était la même pour tous...

\section{L'UE et "l'adaptation " des échelles d'action}

Depuis 2004, l'Agence européenne pour l'environnement (AEE) met en avant la nécessité de disposer de stratégies d'adaptation aux niveaux européen, national, régional et local. L'Union Européenne a donc proposé un « livre vert», le 26 juin 2007, qui examine les éléments de la stratégie d'adaptation à toutes les échelles ${ }^{11}$. Dès l'introduction, ce livre vert reconnaît que l'adaptation est une question complexe car les effets du réchauffement varieront d'une région à l'autre selon la vulnérabilité «physique, le niveau de développement socio-économique, la capacité d'adaptation de la population et du milieu naturel, les services de santé et les mécanismes de surveillance des catastrophes »... La politique européenne d'adaptation préconise aussi la création de processus de gouvernance ${ }^{12}$ vu la multitude d'acteurs : du citoyen jusqu'aux autorités publiques et à l'Union européenne. Les actions doivent être menées « au niveau le plus adapté », doivent être complémentaires et s'appuyer pour cela sur des partenariats. Les compétences entre les États et leurs régions sont réparties de manières très diverses dans les différents pays de l'UE; il faut donc que les mesures prises soient adaptées à la situation nationale. Pour la plupart des mesures d'adaptation, leur mise en œuvre nécessite une participation des autorités nationales, régionales et locales et d'autres autorités (telles que les administrations des bassins versants) ainsi qu'une coordination étroite entre ces instances. Une approche unique de l'adaptation ne convient donc pas car les répercussions des changements climatiques ne s'arrêteront pas aux frontières 
administratives, les changements urbains nécessitent de travailler à l'échelle des agglomérations par exemple. Dans d'autres cas il faudra adopter une vision transfrontière, axée, à partir des bassins fluviaux (les inondations dues au Rhin et à la Meuse), des régions biogéographiques (modèles appliqués à l'arc alpin). Selon l'UE, l'adaptation est pour une large part une question de cohérence politique, de planification et de coordination des actions. Le texte ne va guère plus loin, car cette institution ne peut que soutenir des projets, nécessairement territorialisés, dont le contenu n'est pas totalement de son ressort.

\section{Les entreprises et la loi du marché}

Toutes les entreprises (multinationales comme PME) cumulent les vulnérabilités territoriales, puisque leurs établissements s'inscrivent dans des territoires (la localisation de la matière première décide du lieu d'extraction, le bassin de main d'œuvre du lieu de production, etc. ) et les vulnérabilités du marché. Si une grande entreprise est montrée du doigt sur Internet comme ne respectant pas l'environnement, elle risque un boycott de ses produits par les consommateurs et la dégringolade de ses bénéfices. D'ailleurs, la plupart affiche plus ou moins leur intérêt pour le développement durable. Lafarge, numéro 1 de l'industrie du ciment, par exemple, a créé un partenariat avec WWF en 1998, reconduit en 2005, qui engage la société dans un programme de réduction de ses émissions de CO2 (10\% pour 2010, grâce aux énergies renouvelables) et de restauration des sites dégradés par son activité. Le site Internet d'AREVA annonce que l'entreprise " a fait du développement durable la clef de voûte de sa stratégie industrielle avec la triple ambition d'une croissance rentable, socialement responsable et respectueuse de l'environnement. Son métier : innover pour contribuer à une production d'énergie sans $\mathrm{CO} 2$ et à un acheminement de l'électricité, toujours plus propres, plus sûrs et plus économiques ». Ces annonces ne sont pas incompatibles avec des comportements moins acceptables comme l'utilisation de paradis fiscaux (l'Irlande par exemple pour Veolia), voire de paradis « environnementaux », où les législations sur la pollution sont plus tolérantes. Bien des engagements sont, pour une part, opportunistes ce que résume, malgré lui, le PDG de General Electric avec la formule « la nature a la couleur du billet vert $\gg$.

Mais, en réponse aux exigences des clients, les entreprises sont contraintes de mettre sur le marché des produits plus respectueux de l'environnement. Dans le secteur automobile, Mercedes Benz commercialisera en 2009, la S400 Blue hybrid en attendant la Fcell à hydrogène en 2015. Enfin, les réactions des entreprises, diverses par leur taille (PME/multinationale), leur activité (nucléaire/ extractive...), leur volontarisme « vert», obligent à repenser la dichotomie atténuation/adaptation. Inventer un nouveau moteur qui émet moins de GES est une manière de faire de «l'atténuation » mais si le moteur est utilisé par une pompe pour assécher un marais, c'est alors une action d'« adaptation »...

Progressivement, les complémentarités entre actions d'adaptation et d'atténuation émergent tout comme le fait que l'adaptation ne va pas de soi et a besoin d'accompagnement (financier, administratif, etc.).. Mais la méthode analytique par thème (la biodiversité, voire même une 
espèce, celle du loup, par exemple), par activité ou filière (l'élevage, la gestion forestière, etc.), par lieu (l'arc alpin, le Mercantour,...) exclut d'appréhender la synthèse issue d'un diagnostic territorial.

\section{L'acclimatation des territoires : à problème planétaire, réponse géostratégique}

En quoi consiste donc l'adaptation ? Pour le GIEC il s'agit d'effectuer un « ajustement des systèmes » à la nouvelle donne climatique. Ce terme d'ajustement est trompeur car il signifie «mettre aux bonnes dimensions par réglage, arranger des pièces de façon adéquate par rapport à un objectif ». Or, pour le climat, le dispositif de mise en adéquation recouvre en fait deux réalités distinctes : d'une part, une acclimatation graduelle, progressive à la hausse de température et aux modifications des précipitations (rythmes, intensités, cumuls) et une capacité à encaisser des phénomènes extrêmes d'amplitude nouvelle, jamais enregistrés donc difficiles à imaginer. Enfin, l'objectif (la nouvelle nature du climat en quelque sorte) n'est pas connu dans son intégralité dès maintenant et se modifie en permanence. Le terme d'adaptation ne peut donc s'employer au mieux qu'au pluriel. Et même, il conviendrait de lui substituer un terme mêlant atténuation et adaptation comme par exemple celui d'acclimatation, mot forgé par Buffon en 1775 pour parler des processus de survie des espèces vivantes soumises à des changements progressifs et à des crises. D'ailleurs, le terme est repris dès 1782 pour les groupes humains (conditions de vie et de pensée). Il est donc intéressant dans le cas du changement climatique de revenir à ce mot construit atour du substantif climat et en lien avec lui.

\section{L'acclimatation de chaque territoire = une approche intégrée}

Le futur intègre des évolutions lentes et des événements brutaux. L'acclimatation doit concilier d'une part, des mesures actives prises avant que les impacts ne soient constatés (mesures anticipatoires) en réponse à l'évolution attendue. Des aménagements (construction de digues, enrochements, etc.) vont être effectués pour éviter le recul du trait de côte face à la hausse progressive du niveau de la mer, comme les Hollandais le font depuis cinq siècles au lieu d'immigrer ! Mais, on ne peut éviter des mesures réactionnelles prises lors de crises (mesures correctives) comme celles qui peuvent être envisagées après l'analyse des dégâts et des dommages d'un événement « exceptionnel » (une grande tempête ayant cumulé haute mer et gros coefficient de marée...). Ces mesures publiques et privées pour être compatibles doivent être pour partie planifiées car les mesures anticipées coûtent souvent moins cher que les actions réactives. La planification est également nécessaire pour la prise en compte des intérêts collectifs dans les aménagements successifs. Cette projection dans le futur répond à des choix pour lesquels le savoir-faire existe, d'autant plus que les techniques ont évolué (la pelleteuse a remplacé la pelle pour creuser les canaux, le bulldozer, le panier, pour construire les digues, la pompe électrique s'est substituée au moulin à vent pour évacuer l'eau).

Mais, contrairement à l'image catastrophiste véhiculée par un grand nombre de médias, le changement n'aura pas que des impacts négatifs (température estivale caniculaire, pluie diluvienne et génératrice d'inondations dévastatrices, 
convoitises sur l'eau allant jusqu'aux guerres, réfugiés climatiques par centaines de milliers, etc.). Les revendications des gouvernements des pays riverains ${ }^{13}$ de $1^{\prime}$ Arctique, ont montré les avantages économiques potentiels liés au réchauffement dans « les grands Nord». Ces avantages existent presque partout à condition de choisir de regarder « le verre à moitié plein » : le radoucissement des hivers devrait conduire à une diminution des besoins en chauffage, de nouvelles aires deviendront attractives pour les touristes en été... S'acclimater signifie donc penser les bénéfices issus du changement et tenter de les maximiser. Dans l'agriculture, ce peut être par l'introduction de cultures auxquelles l'allongement de la saison de croissance sera profitable. Moët et Chandon considère que pour le moment "Le réchauffement climatique n'a que des aspects positifs : moins d'acidité, plus de sucre. Les années chaudes ont vu la production des meilleurs millésimes (1964, 1976,1982). Mais dans le futur, il se peut qu'il faille produire ailleurs ». L'entreprise a donc acheté des terres agricoles de versant en Angleterre (Sussex et le Kent) afin d'y cultiver les cépages, qui permettent de produire du vin «méthode champenoise » car la demande explose sur le marché mondial. Pendant que des vignerons catalans investissaient dans les terres nord-pyrénéennes !

Mais, l'analyse activité par activité, enjeu par enjeu ne permet pas d'appréhender la complexité des territoires. Bien des crises sont simplifiées en surdéterminant le climat. La part du changement climatique a par exemple été surévaluée pour l'épidémie de palud en Ouganda en 1994. Une analyse précise montre que durant les trois dernières décennies la température est restée stable.
Mais que l'accroissement de population, suite à l'émigration de populations fuyant les massacres du Rwanda, a conduit à défricher puis cultiver des fonds de vallée autrefois occupés par des marais à papyrus. Comme les papyrus sécrètent à la surface de l'eau un corps gras qui empêche le renouvellement des moustiques, le vecteur n'existait pas tant que les bas fonds étaient des marécages. Pour démêler l'écheveau des facteurs précurseurs, aggravants, déclenchants, il convient de penser plusieurs secteurs simultanément et donc des acteurs très variés et d'échelles différentes. Les acclimatations répondent à une finalité climatique qui s'inscrit dans un contexte plus large, celui que l'on nomme aujourd'hui développement durable. Une mesure pour le climat ne peut accroître une autre forme de vulnérabilité ou participer à la déqualification du territoire. La finalité sociale est donc définie par la synthèse des enjeux sur un territoire. Cette finalité, ce projet collectif est construit par la société humaine qui y habite, travaille, circule, consomme... Un exemple : que choisir pour la baie du Mont saintMichel. Si la finalité est de produire du mouton de pré salé, alors il faut transformer la baie en vasière sous l'eau au rythme des marées, si l'objectif est la production céréalière ou fourragère dans des champs, il convient de stopper l'entrée de la mer et d'endiguer, si la finalité est de maintenir un tourisme patrimonial attiré par un village sanctuaire perché et entouré par les eaux, il faut faire entrer le mer pour créer une île... À chaque option, une décision collective et des investissements ad hoc. Au cours des temps historiques, ces choix successifs ont commandé la poldérisation ou la dépolderisation. Toute l'Histoire montre que les adaptations ne peuvent être que temporaires et réversibles sur le moyen et long termes parce que 
le monde évolue et une solution d'aujourd'hui sera obsolète avec de nouvelles technologies, une démographie modifiée etc. Le développement durable n'est pas écrit, il se construit et reconstruit tous les jours.

Sur une même commune, des décisions d'actions différentes mais compatibles peuvent être prises selon les parcelles, les types d'habitat, les fonctions, etc., depuis « faire face » jusqu'à « valoriser » en passant par « prévenir » $\left(\right.$ tableau $\left.\mathrm{n}^{\circ} 1\right)$.

\section{Les outils des acteurs}

Lorsqu'une collectivité territoriale, y compris l'État, décide de mener à bien un projet d'aménagement et de développement sur son territoire, elle implique de multiples acteurs, qui disposent d'un ensemble d'outils de cinq types différents :

- L'incitation. Elle passe souvent par la fiscalisation sous forme de bonus/malus, de prêt à taux zéro, voire de subventions ou réductions d'impôts sur les coûts. Ce type de mesures est décrété ou voté par les Ètats, les Régions, les collectivités territoriales. En matière d'énergie renouvelable par exemple, le gouvernement espagnol a adopté en 2000 des mesures concernant le prix de rachat de l'électricité issu de l'énergie éolienne si favorable qu'il a entraîné en cinq ans la multiplication par 6 de la puissance installée. Un autre aspect de l'incitation est l'éducation dont les acteurs sont l'État, les collectivités, les ONG, les enseignants, les citoyens, les médias. On pense aux programmes scolaires où l'environnement a fait une entrée remarquée en SVT et géographie.

Tableau 1 : Résumé des différentes Stratégies d'acclimatation

\begin{tabular}{|c|c|c|c|}
\hline ATTITUDES & STRATEGIES & EXEMPLES & TEMPS de mise en œuvre \\
\hline \multirow[b]{2}{*}{ FAIRE FACE } & $\begin{array}{l}\text { (-) Laisser faire } \\
\text { ABANDONNER. }\end{array}$ & $\begin{array}{l}\text { Polders ré-envahis par la mer, } \\
\text { retour aux marais littoraux }\end{array}$ & Immédiat \\
\hline & $\begin{array}{l}(+) \text { Limiter les } \\
\text { pertes } \\
\text { PROTEGER. }\end{array}$ & $\begin{array}{l}\text { Rehausser les digues, bâtir des } \\
\text { épis pour empêcher les inonda- } \\
\text { tions. }\end{array}$ & Plusieurs mois à années \\
\hline \multirow{2}{*}{ PREVENIR } & $\begin{array}{l}\text { (-) Partager les } \\
\text { pertes } \\
\text { ASSURER. }\end{array}$ & $\begin{array}{l}\text { Surtaxer partiellement si risque } \\
\text { élevé mais mutualiser les pertes }\end{array}$ & Plusieurs années \\
\hline & $\begin{array}{l}\text { (+) Délocaliser } \\
\text { hommes et activités } \\
\text { DEPLACER }\end{array}$ & $\begin{array}{l}\text { Raser des maisons et recons- } \\
\text { truire ailleurs. }\end{array}$ & Plusieurs mois \\
\hline \multirow{2}{*}{ VALORISER } & $\begin{array}{l}(-) \text { Creer de nouvel- } \\
\text { les activités } \\
\text { MUTER. }\end{array}$ & $\begin{array}{l}\text { Cultiver la vigne en Normandie } \\
\text { au lieu des pommiers. }\end{array}$ & Plusieurs années \\
\hline & $\begin{array}{l}(+) \text { Augmenter les } \\
\text { gains } \\
\text { S'ENRICHIR. }\end{array}$ & Innover, Investir & Plusieurs années \\
\hline
\end{tabular}


De nombreux sites Internet proposent aussi des pages d'autoévaluation de sa propre empreinte écologique, d'autres mobilisent les internautes pour mener des actions coup de poing comme ce fut le cas le mardi 23 octobre 2007 entre 19 h 55

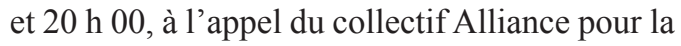
planète (éteindre toute lumière), etc.

- La réglementation. C'est là encore l'État, les collectivités territoriales qui fixent des politiques publiques grâce à des documents de zonage opposables ou non. Citons en France pour les villes le PADD ou Plan d'aménagement durable du territoire, Instauré par la loi SRU (Loi Solidarité et Renouvellement Urbain), document, non opposable, qui fixe les objectifs en matière d'habitat, de développement économique, de loisirs, de déplacement, d'équipement commercial, etc. Les PPR qui délimitent les zones exposées aux risques, dites « zones de danger », en y interdisant tout type de construction, d'ouvrage, d'aménagement ou d'exploitation, se traduisent souvent directement dans les PLU. Cette réglementation peut aller jusqu'à l'expulsion des zones « rouges » et donc à des déplacements de résidences ou de lieux d'activités. Dans l'Aude, une trentaine de maisons non protégées par des digues et situées dans un village sinistré par deux fois ont été rachetées par l'État pour être détruites dans le cadre du programme de prévention des inondations. Comme la loi Barnier impose aussi d'informer, d'autres acteurs entrent en jeu avec le volet communication sur les risques. Il touche tout un chacun lors de la cession d'un bien immobilier, par exemple.

- L'innovation. Les acteurs principaux du monde de l'innovation sont des chercheurs et des inven- teurs, des managers d'entreprise, des ingénieurs. Ils peuvent répondre à de nouveaux besoins ou à des processus plus « économes », plus « écologiques », etc. Depuis 1967, l'innovation fait partie du bagage des futurs ingénieurs de l'École des mines de Paris qui reçoivent des enseignements sur le sujet. De leur côté, certaines banques (AR\&D par exemple) aident à la création d'entreprises mettant au point des prototypes de nouveaux produits industriels issus d'inventions récentes, Ce fut le cas des serres pilotes alimentées par électricité photovoltaïque et pompant l'eau de mer pour faire de l'agriculture en plein désert. D'autres entreprises comme BP investissent dans de nouvelles technologies : depuis 2008, la centrale de Moura au sud du Portugal fonctionne grâce à 350000 panneaux solaires installés sur 114 hectares. Tous les constructeurs automobiles cherchent à produire la voiture électrique de série la plus performante et la moins chère...

- La réparation dont les acteurs majeurs sont les employés des secteurs des assurances puisque le législateur français a mis essentiellement à la charge des compagnies ou mutuelles d'assurances des biens sinistrés l'indemnisation des conséquences matérielles des risques naturels, dont ceux liés au climat. Les deux tempêtes de décembre 1999 ont coûté environ 7 milliards d'euros. Quant aux entreprises, leurs interventions impliquent toute une série d'acteurs depuis les ouvriers jusqu'aux directeurs, en passant par les commerciaux, les ingénieurs, etc. Voire des architectes. À Nîmes, la restauration des infrastructures des quartiers endommagés par l'inondation d'octobre 1988, ainsi que l'ensemble des mesures du plan CADEREAU a concerné près d'une vingtaine d'entreprises de la région, 
principalement du BTP.

- La gestion de crise relève du maire, du préfet de département, du préfet de zone et de région (plan ORSEC ou d'Organisation de la Réponse de SÉcurité Civile,), du ministre de l'Intérieur (arrêté de Catastrophe naturelle depuis 1982) puisque la loi de modernisation de la sécurité civile d'août 2004 énonce en préambule que «la protection des populations compte parmi les missions essentielles des pouvoirs publics ». Mais elle concerne aussi les services opérationnels permanents, les services départementaux d'incendie et de secours (SDIS), les services médicaux d'urgence, les médias, les ONG locales et les citoyens (résidents, commerçants, touristes, employés non résidents, etc.).

L'efficacité des actions passe par la mobilisation de nombreux acteurs de cultures différentes donc c'est une affaire de reconnaissance de chacun en tant qu'acteur, d'écoute réciproque, de controverses, de conflits, d'arbitrages, bref de gouvernance. L'environnement semble d'ailleurs jouer un rôle « d'avant garde » dans la modernisation de ces formes de démocratie, puisque coexistent des modes d'intervention traditionnels de l'État - de type « command and control » (Interventions, réglementation, sanction) et des formes de participation à géométrie variable selon la nature des problèmes en jeu et les contextes ${ }^{14}$.

\section{Deux dispositifs de mobilisation des acteurs : une question de gouvernance}

En France, le Plan Climat (2004 puis 2006), la « Charte pour l'environnement» proposée à la signature de tous les maires de villes françaises en $2007^{15}$, ont reconnu que les collectivités locales détiennent les leviers incontournables d'actions contre les changements climatiques. Bien qu'éparse et peu contraignante ${ }^{16}$ la législation attribue une place majeure au Plan climat territorial qui suppose d'établir, en concertation avec les acteurs : un bilan carbone, un diagnostic des vulnérabilités aux changements à venir et un plan d'action pour la collectivité (bâtiments, urbanisme, transports, développement économique, éducation et sensibilisation...). Or, sur 21 Plans climats territoriaux étudiés en 2007 par la MIES, un sur deux seulement comporte une analyse de vulnérabilité du territoire ! Serait-ce que les élus préfèrent l'atténuation à l'adaptation ? Certes, il peut leur sembler que l'adaptation est naturelle et que la cause de la planète est un enjeu valorisant. Sans doute pensent-ils aussi que, malgré les progrès récents, l'échelle spatiale, les horizons à 50 ans ou plus, et les fourchettes larges des scenarii ne conviennent pas à leur action. Mais, surtout ils considèrent que l'Ademe, la MIES et le ministère chargé du développement durable n'ont mis aucun outil à leur disposition pour les aider. Le discours de ces organismes (Faisons vite ça chauffe !) ainsi que ceux d'associations comme R.A.C., Énergies-Cité, est consacré d'ailleurs pour l'essentiel à la limitation des émissions de GES. Au final, le fonctionnement français, pays très centralisé (la décentralisation a une vingtaine d'années) a du mal à fonctionner autrement que sous forme up-down (le Glo-cal), qui privilégie le point de vue de minorités « averties » alors que le « zoom » des adaptations du global au local suppose du «sur-mesure».

Pendant, ce temps, les Pays-Bas particulièrement motivés en raison de la configuration de leur 
territoire très plat (Amsterdam est à -2m d'altitude) et très peuplée donc très vulnérable à une élévation du niveau de la mer et aux inondations de la Meuse et du Rhin, ont fait des choix en faveur de l'adaptation. Leur Plan climat national a été décliné à toutes les échelles. Après une analyse fine (quartier par quartier puis parcelle par parcelle, les microsites ${ }^{17}$ en quelque sorte), des aléas, des enjeux et des risques, des réponses ont été adoptées : rehaussement de certaines digues, renforcement des écluses, multiplication des stations de pompage, création de brises lames insulaires, surélévation de bâtiments patrimoniaux, re-création de champs d'épandage, évacuation de pâtés de maisons, etc. Un calendrier des travaux et de financement a été mis au point en concertation avec tous les acteurs (résidents, entreprises, associations locales, aménageurs, agriculteurs, commerçants, etc.). La télévision a même retransmis en direct des débats. Ces initiatives commencent à créer des emplois ici tout en enlevant ailleurs, elles permettent d'innover, de repenser les solidarités en « vivant avec » et non « contre » le changement de climat, comme on le disait avec la mer. C'est une autre démocratie qui se met en place, respectant la mémoire collective, les représentations. Cette gouvernance offre d'ailleurs une solution alternative au problème du scepticisme face à la démocratie réduite aux élections que traduit l'absentéisme ou le vote sanction. Les habitants participent ainsi à des formes de démocratie de proximité ${ }^{18}$ qui reconstruit le lien social autour de perspectives communes. Il est vrai que leur culture les y prépare mieux.

Il n'y a donc aucune fatalité. Construire des passerelles entre les univers qui ont souvent tendance à s'ignorer suppose de remettre l'habitant, l'en-

Tableau 2 : Résumé des deux pôles de méthodologie de la décision

\begin{tabular}{|c|c|c|}
\hline & CENTRALISEE & DECENTRALISEE \\
\hline Problème & connu y compris solutions & $\begin{array}{l}\text { recadrer en contextualisant (lieu, his- } \\
\text { toire) }\end{array}$ \\
\hline Méthode diagnostic & modèles, virtuel & concret, expérimentation \\
\hline Moyens & $\begin{array}{l}\text { rechercher normes, lois univer- } \\
\text { selles }\end{array}$ & $\begin{array}{l}\text { raisonner sur processus territorialisés } \\
\text { spécifiques }\end{array}$ \\
\hline Acteurs & $\begin{array}{l}\text { grandes instances inter ou multina- } \\
\text { tionales. État }\end{array}$ & tous acteurs y compris quidam \\
\hline Politique & Sectorielle & $\begin{array}{l}\text { intégré (global au sens français du ter- } \\
\text { me) }\end{array}$ \\
\hline Échelles & Planète, groupe d'États, Ėtats & territoire pertinent \\
\hline Processus & technico-administratif & $\begin{array}{l}\text { concertation acceptation par gouver- } \\
\text { nance }\end{array}$ \\
\hline Objectif & $\begin{array}{l}\text { survie planète, survie de l'huma- } \\
\text { nité }\end{array}$ & $\begin{array}{l}\text { "viabilité » de chaque territoire, vivre } \\
\text { ensemble }\end{array}$ \\
\hline Pas de temps & 2049,2099 , horizons lointains & horizon proche \\
\hline $\begin{array}{l}\text { Caractéristiques des } \\
\text { actions }\end{array}$ & $\begin{array}{l}\text { Résultats invisibles par un individu, } \\
\text { irréversible quelque soit les inno- } \\
\text { vations }\end{array}$ & $\begin{array}{l}\text { résultats visibles et réversibles avec évolu- } \\
\text { tion techniques, démographiques, etc. }\end{array}$ \\
\hline
\end{tabular}


treprise au cœur du système de décision par une procédure multi acteurs (appelée décentralisée dans le tableau n ${ }^{\circ}$ 2). C'est un long apprentissage réciproque. «Il aura fallu dans les domaines traditionnels de la délibération publique plusieurs siècles pour que les règles du débat soient transformées en institutions durables, et la complexité inhérente aux problèmes d'environnement rend cette institutionnalisation dans les nouveaux espaces du débat encore plus difficile, ne serait ce qu'en raison de leurs dimensions transnationales $\rangle^{19}$. De nombreux échanges, parfois conflictuels, des aller et retour sont nécessaires avant tout choix. La lenteur du processus est compensée par son caractère motivant et son efficacité. De toute façon, toute adaptation « durable » ne peut reposer sur des décisions prises dans l'urgence. $\mathrm{Si}$ la recherche du consensus est souhaitable, elle n'exclut d'ailleurs pas in fine un arbitrage.

La culture du risque existe depuis cinq-six siècles au moins dans certains pays, où le maintien d'un niveau de vie élevé sur un territoire exigu a supposé un effort concerté et gigantesque de tous (urbains, agriculteurs, éleveurs, pêcheurs, commerçants, mariniers, industriels, etc.). Dans d'autres pays, ces savoirs et savoir-faire n'existent que régionalement pour certains risques bien localisés (avalanches en montagne, inondations de fond de vallée à crue " méditerranéenne ", envasement de fond de baie sur des côtes à marée, etc.). Le passage par la territorialisation est donc obligatoire.

Le problème de l'action face aux changements liés au climat gagne en objectivité en croisant les subjectivités de chacun, à condition qu'il soit mis en situation d'acteur. Selon Bernard Manin, «la décision légitimen'est pas la volonté de tous mais celle qui résulte de la délibération de tous». Dans le cas de solutions «parachutées », les décisions risquent d'être mal adaptées au contexte local, (naturel, économique, historique, culturel, social) voire rejetées faute d'être comprises. C'est donc les liens entre des niveaux jugés emboîtés qu'il faut reconstruire.

\section{Conclusion générale}

L'acclimatation n'a pas de raison d'être si effectivement le système planétaire bascule vers quelque chose d'inconnu. Il est impossible d'imaginer des solutions à un emballement. Et, si comme le dit Pachaury (Président du GIEC et prix Nobel) «nous n'avons que 7 ans... $»^{20}$, attention à la tentation de baisser les bras ! Communiquer pour mobiliser est certainement plus efficace.

Les problèmes posés aux sociétés ne sont pas de même nature que des problèmes de mathématiques ou de physique ayant une solution unique. En sciences sociales, certaines solutions, qui rencontrent des obstacles à leur application, peuvent aggraver le problème. Plutôt que de chercher LA solution, il est nécessaire d'explorer le problème, mal posé, pour le reformuler : ici, «s'acclimater à », « vivre avec » et non « lutter contre».

Quant aux attitudes possibles face au changement, il ressort de leur analyse que :

- L'atténuation suppose que tous les individus et tous les groupes humains ont et auront un comportement vertueux. C'est une utopie car il est impossible de faire respecter par tous des règlements dont l'application serait contrôlée par une instance mondiale coercitive. 
- L'acclimatation est moins ambitieuse, elle implique chacun dans son propre voisinage immédiat, son territoire. Elle se construit à partir de la conciliation de multiples égocentrismes, voire égoïsmes. Pragmatique, elle envisage les individus, les groupes sociaux comme ils sont et non comme ils devraient être, y compris le syndrome NIMBY. Pour des raisons pas toutes très « nobles », les micro-acteurs participent donc consciemment à une macro-action de réduction du risque futur.

Comme, les territoires sont et seront inégaux face aux risques, la justice sociale (territoriale ?) impose des solidarités sous forme de transferts de technologies, de capitaux, etc. vers les pays pauvres, vers les régions les plus exposées. D'ores et déjà les pays riches n'honorent pas leurs engagements financiers quant à la réduction de moitié de la pauvreté dans le monde (objectif du millénaire), alors qu'en sera-t-il après la crise financière et économique de la fin 2008 ? Parviendra-t-on à «Action locale, solidarité globale »?

\section{$R \cdot E ́ \cdot F \cdot E \cdot R \cdot E \cdot N \cdot C \cdot E \cdot S$}

AGARWAL (Anil), « Pour un juste calcul des responsabilités », La Recherche, n²43, 1992, pp. 610-613.

ARNOULD (Paul) 2007 Entreprises et développement durable : une relation ambiguë, La Revue des Deux Mondes : Le développement durable, une idéologie?

BREDIF( Hervé) 2008, La qualité comme moyen de repenser le développement durable d'un territoire, Espaces-temps, Textuel, sur http:// espacestemps.net/document5213.html

BRÉDIF (Hervé), Le vivant, les hommes et le territoire, Essai de biogéopolitique, thèse de doctorat, deux tomes, 2004.

BADIE (Bertrand), Un monde sans souveraineté, Fayard, Paris, 1999, 306 p.

DAHAN DALMEDICO (Amy), GUILLEMOT (Hélène), «Changement climatique : Dynamiques scientifiques, expertise, enjeux géopolitiques », ScienceDirect, 2006, 21p. http://math. unipa.it/ grim/dott_HD_MphCh/Dahan_Changement $\% 20$ climatique_06.pdf

DENIS (Benjamin), « La gouvernance mondiale de la préservation de l'environnement : acteurs non étatiques et processus politique international lié au réchauffement de la planète ", Cahiers d'anthropologie du droit, 2005, pp. 66-122.

GODARD (Olivier) et HOMMEL (Thierry), Les multinationales, un enjeu stratégique pour l'environnement et le développement durable ? Revue internationale et stratégique 2005- $4 \mathrm{~N}^{\circ} 60$, pp. 101-112

LAMARRE (Denis) Climat et risques, nouvelles approches, 2008, Lavoisier, 170 p.

LEFEVRE (Mathias), « Les Firmes multinatio- 
nales face au risque climatique : sauver le capital en sauvant la terre », VertigO, vol. 5, n², septembre 2004, 13p. disponible sur : http:/www. vertigo.uqam.ca/vo15no2/art6vol5no2/vertigovol5no2_lefevre.pdf

LE PRESTRE (Philippe), Protection de l'environnement et relations internationales : les défis de l'écopolitique mondiale, Armand Colin, Paris, 2005, 466 p.

MANIN, (Bernard), «Volonté générale ou délibération? Esquisse d'une théorie de la délibération politique», Le Débat, 1985, 33, pp.72-94.

MENDELSOHN (Robert) et NEUMANN (James), The impact of climate change on the United States Economy, Cambridge university Press, 1999, $331 \mathrm{p}$.

ROQUEPLO (Philippe), « L'effet de serre est-il politiquement gérable? », Futuribles, n²24, 1997, pp.17-32.

ROSANVALLON (Pierre) La légitimité démocratique : Impartialité, réflexivité, proximité, Seuil 2008, 368 p

ROSENAU (James Nathan), Governance without government : Order and Change in World Politics, Cambridge University Press, Cambridge, 1992, 311 p.

SMITH Joel, BHATTI (Neeloo), MENZHULIN (Gennady), BENIOFF (Ron), BUDYKO (Mikhail), CAMPOS (Max), JALLOW (Bubu) et RIJSBERMAN (Franck), Adapting to climate change-assessments and issues-, 1996, Springer, $476 \mathrm{p}$

STRANGE (Susan), The retreat of the State: the Diffusion of Power in the World Economy, Cambridge University Press, Cambridge, 1996, 244 p.

VALANTIN (Jean-Michel), Menaces climatiques sur l'ordre mondial, Lignes de repères, Paris,
2005, $158 \mathrm{p}$.

VALANTIN (Jean-Michel), Écologie et gouvernance mondiale, Autrement Frontières, Paris, 2007, $150 \mathrm{p}$. 
N

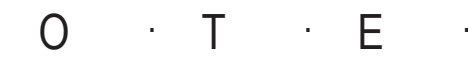

S

1. Le GIEC a confirmé le 2 février 2007 que la probabilité que le réchauffement climatique soit dû à l'activité humaine est supérieure à $90 \%$. Cf. http:// www.ipcc.ch/SPM2feb07.pdf.

2. Lutte signifie "combat, action contre quelqu'un, quelque chose". Dans le cas du climat, cet ennemi est invisible et les menaces comme les négociations sont sans effet sur lui.

3. GIEC, Rapport du groupe de travail I, résumé à l'intention des décideurs, 2007, http://www.ipcc.ch/ pdf/assessment-report/ar4/wg1/ar4-wg1-spm-fr.pdf.

4. Il s'agit des Article 10 (b) : Toutes les parties «élaborent, appliquent, publient et mettent régulièrement à jour des programmes nationaux et, là où il y a lieu, régionaux, contenant des mesures destinées à atténuer les CC et des mesures destinées à faciliter une adaptation appropriée à ces changements ", 10 (b) (i) : «Ces programmes devraient concerner notamment les secteurs de l'énergie, des transports et de l'industrie ainsi que l'agriculture, la foresterie et la gestion des déchets. En outre, les technologies d'adaptation et les méthodes visant à améliorer l'aménagement de l'espace permettraient de mieux s'adapter aux changements climatiques », 10 (b) (ii) : Les Parties communiquent des informations sur leur action au titre du Protocole, y compris sur leurs mesures d'adaptation et de l'article 12.8: « La Conférence des Parties (...) veille à ce qu'une part des fonds provenant d'activités certifiées soit utilisée pour (...) aider les pays en développement Parties qui sont particulièrement vulnérables aux effets défavorables des changements climatiques à financer le coût de l'adaptation ".

5. In "Changement climatique : Dynamiques scientifiques, expertise, enjeux géopolitiques" de Amy Dahan Dalmedico et Hélène Guillemot, Sociologie du Travail, Volume 48, Issue 3, July-September 2006, Pages 412-432.

6. Le principe de précaution officiellement entériné en 1992 dans la convention de Rio n'a pas de définition universellement admise. Celle de la loi française de 1995 est : "l'absence de certitudes, compte tenu des connaissances scientifiques et techniques du moment, ne doit pas retarder l'adoption de mesures effectives et proportionnées visant à prévenir un risque de dommages graves et irréversibles [...] à un coût économiquement acceptable ».

7. Le prix Nobel de chimie Paul Crutzen propose d'annuler l'effet des GES en injectant à environ $20 \mathrm{~km}$ d'altitude, de gigantesques quantités de soufre ou de sulfure d'hydrogène transporté par des ballons pour un coût de 25 milliards de dollars (revue Climatic Change).

8. Le territoire est défini en géographie comme un espace habité et exploité donc approprié et administré où se développe une identité, une socialisation qui permet au groupe d'exister.

9. http://www.hmtreasury.gov.uk/independent reviews/stern_review_economics_climate_change/ stern_review_report.cfm

10. http://unfecc.int/adaptation/sbsta_agenda_item adaptation/items/3633.php.

11. Livre vert européen : «Adaptation au changement climatique en Europe », COM (2007) 354 final, juin 2007.

12. En 1992, dans Governance without Government, James Rosenau caractérise la gouvernance comme « un ensemble de mécanismes de régulation existant dans une sphère d'activité donnée, et qui fonctionnent, alors même qu'ils n'émanent pas d'une autorité officielle. En 1995, la Commission sur la gouvernance mondiale désigne la gouvernance comme « un processus continu de coopération et d'accommodement entre des intérêts divers et conflictuels. Elle inclut les institutions 
officielles et les régimes dotés de pouvoirs exécutoires tout aussi bien que les arrangements informels sur lesquels les peuples et les institutions sont tombés d'accord ou qu'ils perçoivent être de leur intérêt ".

13. Canada, États-Unis, Russie, Norvège, Islande et Danemark (via le Groenland).

14. Olivier Godard, "Stratégies industrielles et convention d'environnement. De l'univers stabilisé aux univers controversés », INSEE méthode 1993, Paris. 15. Cf.l'article «Lutte contreleréchauffementclimatique: la Commission européenne lance la « Convention des maires », 30 janvier 2008, http://www.maire-info.com/ article.asp?param=9264\&PARAM2=PLUS.

16. La LAURE et les PDU, la loi POPE de juillet 2005, Les contrats de plan Etat-Région dont la «neutralité carbone » de la dernière génération (2007), Les Plans climat territoriaux et même le projet de loi Grenelle ! 17. W. Hucy et al., 2005, in N. Mathieu et Y. Guermond, La ville urable, Paris Cemagref, pp. 237-260.

18. P. Rosanvallon.

19. G. Majone, «Décisions publiques et délibération », in Revue française de science politique, vol $44, n^{\circ} 4$, août 1994.

20. In Le Monde, 7 juillet 2008.

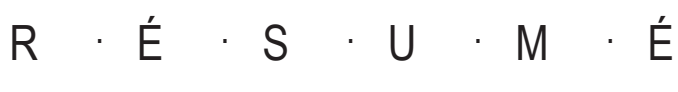

Le réchauffement climatique implique une réaction combinant l'atténuation de l'amplitude du réchauffement et l'adaptation pour minimiser les effets néfastes ou utiliser au mieux les opportunités bénéfiques. Mais ces actions combinées supposent un projet commun faisant intervenir un grand nombre d'acteurs. Au cours des décennie 1980 et 1990, la réponse a été internationale et a privilégié les actions d'atténuation. Mais après le rapport Stern, sont mises en avant les inégalités des territoires face au risque. L'Union européenne cherche alors à définir les échelles d'action pertinentes. Ensuite, progressivement, est mise en place une réflexion sur les stratégies d'adaptation différenciée des territoires.

Global warming implies a combined reaction : mitigation to reduce greenhouse gas emissions and to enhance sinks aimed at reducing the extent of global heating and adaptation which involves taking action to minimize the negatives effects and to optimize new opportunities. At the end of the XX th century, the answer was international and mitigation was the only way. After the Stern Review adaptation appears as a necessary strategy to complement mitigation efforts because natural and human systems are not equal against risks. Then, gradually, adaptation initiatives and measures are now expanding to operate at many different interlinked scales. 
